Journal of Computer Science 7 (5): 715-722, 2011

ISSN 1549-3636

(C) 2011 Science Publications

\title{
Accurate Orthogonal Circular Moment Invariants of Gray-Level Images
}

\author{
Khalid Mohamed Hosny \\ Department of Computer Science, Community College, \\ Najran University, Najran, P.O. Box 1988, Saudi Arabia
}

\begin{abstract}
Problem statement: Orthogonal circular moments of gray level images such as Zernike, pseudo Zernike and Fourier-Mellin moments are widely used in different applications of image processing, pattern recognition and computer vision. Computational processes of these moments and their translation and scale invariants still an open area of research. Approach: a unified methodology is presented for efficient and accurate computation of orthogonal circular moment invariants. The orthogonal circular moments and their translation and scale invariants are expressed as a linear combination of radial moments of the same order in polar coordinates, where the later moments are accurately computed over a unit disk. A new mapping method is proposed where the unit disk is divided into non-overlapped circular rings; each of these circular rings is divided into a number of circular sectors of the same area. Each circular sector is represented by one point in its centre. The total number of input Cartesian image pixels is equal to the number of mapped circular pixels. Results: The implementation of this method completely removes both approximation and geometrical errors produced by the conventional methods. Numerical experiments are conducted to prove the validity and efficiency of the proposed method. Conclusion: A unified methodology is presented for efficient and accurate computation of orthogonal circular moment invariants.
\end{abstract}

Key words: Radial moments, Zernike moments, pseudo Zernike moments, Fourier-Mellin moments, translation invariants, scaling invariants, accurate computation

\section{INTRODUCTION}

Orthogonal circular moments of gray level images such as Zernike, pseudo Zenike and Fourier-Mellin are used to represent images with minimum amount of information redundancy. Based on this attractive property, these orthogonal moments are widely used in different image processing and pattern recognition applications. See for examples (Abandah and Anssari, 2009, Radhika et al., 2010, Ismail et al., 2010).

Despite of this fact, the conventional approach for computing these circular moments and their invariants encounter two major problems. These problems are the lack of accuracy and the very time-consuming computational processes.

Different methods were proposed to overcome these problems for each kind of these moments. Remarkable works are presented to efficiently compute Zernike moments (Xin et al., 2007; Hosny, 2008; 2010a; 2010b) and pseudo Zernike moments (Chong et al., 2003a; Al-Rawi, 2010) Another group of works presents efficient computation of orthogonal FourierMellin moments (Papakostas et al., 2007; Hosny et al., 2011; Walia et al., 2011).
The variety of these methods motivates us to propose a unified efficient method for accurate computation of orthogonal circular moments of binary as well as gray level images.

The orthogonal circular moments are rotationally invariant by nature. This attractive property enables these moments to play an essential role in the invariant pattern recognition tasks. Invariance with respect to other geometric transformation such as translation and scaling could be achieved through the image normalization method. The literature of moment computation has two works that are dealing with translation invariants of Zernike moments (Chong et al., 2003b) and the scale invariants of pseudo Zernike moments (Chong et al., 2003c). Unfortunately, no work is available for translation and scaling invariants of Fourier-Mellin moments.

This study proposes a new unified method for efficient and highly accurate computation of orthogonal circular moments for binary and gray-level images.

The proposed method is extended to accurately compute both translation and scale moment invariants. The proposed method consists of two major steps. In the first step, highly accurate radial moments are 
computed in polar coordinates. In the second step, all aforementioned circularly moments and their invariants are computed as linear combinations of the radial moments. A number of numerical experiments are conducted where the experimental results clearly show the efficiency of this proposed method.

The rest of the study is organized as follows: An overview of the orthogonal circular moments of images is presented. The proposed method is described in the section of material and methods. Conducted numerical experiments are described and the obtained results are analyzed in the section of results. It followed by a discussion and Conclusion. An acknowledgement of the financial support is presented. Finally, a list of recent references is presented.

Circular moments of images: Orthogonal circular moments of an image are defined over a unit disk in polar coordinates. In this subsection, a brief overview of orthogonal Zernike, pseudo Zernike and FourierMellin moments is presented.

Zernike moments: The complex two-dimensional Zernike moments of order $\mathrm{p}$ and repetition $\mathrm{q}$ are defined as follow:

$$
\begin{aligned}
& \mathrm{Z}_{\mathrm{p}, \mathrm{q}}=\frac{\mathrm{p}+1}{\pi} \times \\
& \int_{0}^{2 \pi} \int_{0}^{1} \frac{\mathrm{V}_{\mathrm{p}, \mathrm{q}}(\mathrm{r}, \theta)}{\mathrm{f}}(\mathrm{r}, \theta) \mathrm{rdr} \theta
\end{aligned}
$$

where, $\mathrm{p}=0,1,2,3, \ldots \ldots \infty$ and $\mathrm{q}$ is positive integer selected according to the conditions: $(\mathrm{p}-\mathrm{q})=$ even and $\mathrm{q} \leq \mathrm{p}$.

Zernike moments with negative values of repetition $q$ are computed using complex conjugate from the relation $\mathrm{Z}_{\mathrm{p},-\mathrm{q}}=\overline{\mathrm{Z}_{\mathrm{p}, \mathrm{q}}}$. Zernike functions $\mathrm{V}_{\mathrm{pq}}(\mathrm{r}, \theta)$ forms a complete set of complex-valued functions which enable the direct computation of inverse transform.

Pseudo Zernike moments: The complex twodimensional pseudo Zernike moments of order $\mathrm{p}$ and repetition $\mathrm{q}$ are defined as follows:

$$
\begin{aligned}
A_{p, q} & =\frac{p+1}{\pi} \times \\
& \int_{0}^{2 \pi} \int_{0}^{1} \overline{W_{p, q}(r, \theta)} f(r, \theta) r d r d \theta
\end{aligned}
$$

where, $\mathrm{p}=0,1,2,3, \ldots \ldots \infty$ and $\mathrm{q}$ is non-negative integer defined according to the condition $\mathrm{q} \leq \mathrm{p}$.
Pseudo Zernike moments with negative values of repetition $q$ are computed from those of positive values by using the relation, $A_{p,-q}=\overline{A_{p, q}}$. Pseudo Zernike functions, $W_{p q}(r, \theta)$, are a complete set of complexvalued orthogonal functions.

Fourier-Mellin moments: Orthogonal Fourier-Mellin moments of order $\mathrm{p}$ and repetition $\mathrm{q}$ are defined as follows:

$$
\begin{aligned}
\mathrm{F}_{\mathrm{p}, \mathrm{q}} & =\frac{\mathrm{p}+1}{\pi} \times \\
& \int_{0}^{2 \pi} \int_{0}^{1} \mathrm{Q}_{\mathrm{p}}(\mathrm{r}) \mathrm{e}^{-\hat{\mathrm{i} q} \theta} \mathrm{f}(\mathrm{r}, \theta) \mathrm{rdrd} \theta
\end{aligned}
$$

where, $\hat{i}=\sqrt{-1}, p=0,1,2,3, \ldots \ldots \infty$ and $q$ is non-negative integer. Similar to Zernike and pseudo Zernike, Fourier-Mellin moments of negative repetition are obtained directly from that of positive repetition.

Central radial moments: Central radial moments of order $\mathrm{p}$ with repetition $\mathrm{q}$ are defined as follows (Chong et al., 2003b):

$$
\hat{\mathrm{R}}_{\mathrm{p}, \mathrm{q}}=\sum_{\mathrm{m}-0}^{\mathrm{u}} \sum_{\mathrm{n}=0}^{\mathrm{v}}\left(\begin{array}{l}
\mathrm{u} \\
\mathrm{m}
\end{array}\right)\left(\begin{array}{l}
\mathrm{u} \\
\mathrm{n}
\end{array}\right)(-\mathrm{A})^{\mathrm{m}}(-\overline{\mathrm{A}})^{\mathrm{n}} \mathrm{R}_{\alpha, \beta}
$$

Where:

$$
\begin{aligned}
& \mathrm{u}=(\mathrm{p}+\mathrm{q}) / 2 \mathrm{v}=(\mathrm{p}-\mathrm{q}) / 2 \\
& \alpha=(\mathrm{u}-\mathrm{m})+(\mathrm{v}-\mathrm{n}) \\
& \beta=(\mathrm{u}-\mathrm{m})-(\mathrm{v}-\mathrm{n}) \\
& \mathrm{A}=\mathrm{x}_{0}-\hat{\mathrm{i}} \mathrm{y}_{0}, \overline{\mathrm{A}}=\mathrm{x}_{0}+\hat{\mathrm{i}} \mathrm{y}_{0}
\end{aligned}
$$
follows:

The centriod of the input image is defined as

$$
\begin{array}{r}
x_{0}=\frac{\sum_{i=1}^{N} \sum_{j=1}^{N} x_{i} f\left(x_{i}, y_{j}\right)}{\sum_{i=1}^{N} \sum_{j=1}^{N} f\left(x_{i}, y_{j}\right)} \\
y_{0}=\frac{\sum_{i=1}^{N} \sum_{j=1}^{N} y_{j} f\left(x_{i}, y_{j}\right)}{\sum_{i=1}^{N} \sum_{j=1}^{N} f\left(x_{i}, y_{j}\right)}
\end{array}
$$

Scale invariants of radial moments: Scale invariants of radial moments of order $\mathrm{p}$ with repetition $\mathrm{q}$ are defined as follows Chong et al., 2003c): 
$\hat{\mathrm{R}}_{\mathrm{p}, \mathrm{q}}=\mathrm{R}_{\mathrm{p}, \mathrm{q}} /\left(\mathrm{R}_{00}\right)^{\frac{\mathrm{p}}{2}+1}$

\section{MATERIALS AND METHODS}

Orthogonal circular moments are defined in polar coordinates over a unit disk. Zernike, pseudo Zernike and Fourier-Mellin moments and their translation and scale invariants could be represented as a linear combination of radial moments of the same order or less. A generic formula of the two-dimensional circular moments and their invariants of order $\mathrm{p}$ with repetition $\mathrm{q}$ is defined as follows:

$$
\mathrm{C}_{\mathrm{p}, \mathrm{q}}=\frac{\mathrm{p}+1}{\pi} \sum_{\mathrm{k}=\mathrm{T}}^{\mathrm{p}} \mathrm{B}_{\mathrm{pqk}} \mathrm{R}_{\mathrm{k}, \mathrm{q}}
$$

where, $\mathrm{p}=0,1,2,3, \ldots \ldots \infty$ and $\mathrm{q}$ is positive integer. The different values of the parameter $\mathrm{T}$ and the coefficient matrix $\mathrm{B}_{\mathrm{pqk}}$ of the aforementioned circular moments are showed in Table 1.

Highly accurate radial moments: It is clear that, the computational process of radial moments, $\mathrm{R}_{\mathrm{kq}}$, is the core part in the whole computational process of these orthogonal circular moments and their invariants. Radial moments of order $\mathrm{k}$ with repetition $\mathrm{q}$ are defined in polar coordinates over a unit disk as follows:

$$
\mathrm{R}_{\mathrm{kq}}=\int_{0}^{2 \pi} \int_{0}^{1} \mathrm{r}^{\mathrm{k}} \mathrm{e}^{-\hat{\mathrm{i}} q \theta} \mathrm{f}(\mathrm{r}, \theta) \mathrm{rdr} \mathrm{d} \theta
$$

where, $\hat{\mathrm{i}}=\sqrt{-1}, \mathrm{k}=0,1,2,3, \ldots \ldots \infty$ and $\mathrm{q}$ is any positive or negative integer. For input image of size $\mathrm{N} \times \mathrm{N}$ as depicted in Fig. 1, a new image mapping is proposed where all of the required computational processes are done in the polar coordinates. This new mapping method is a modification of the method described by Hosny et al. (2011) where the total number of input image pixels is equal to the total number of mapped circular sector pixels as depicted in Fig. 2. The new mapping method is very simple and could be achieved by applying the following steps:

- The unit disk is divided into, $\mathrm{N} / 2$, concentric, nonoverlapped circular rings

- Each circular ring is divided into,(8i+4), circular sectors where $\mathrm{i}=0,1,2, \ldots ., \mathrm{N} / 2-1$ and $\mathrm{i}=0$ refers to the innermost circular ring

- The different values of the angle $\theta$ could be created by using the following pseudo-code:

$$
\begin{aligned}
& \text { for } \mathrm{i}=0 \text { to } \mathrm{N} / 2-1 \\
& \text { for } \mathrm{j}=0 \text { to }(8 \mathrm{i}+4)-1 \\
& \theta_{i, j}=2 \pi(\mathrm{j}+0.5) /(8 \mathrm{i}+4) \\
& \text { endfor }
\end{aligned}
$$

endfor

Table 1: Circular moments and their coefficients

\begin{tabular}{lll}
\hline & Coefficient matrix, $\mathrm{B}_{\mathrm{pqk}}$ & Parameter T \\
\hline & $\frac{(-1)^{\left(\frac{\mathrm{p}-\mathrm{k}}{2}\right)\left(\frac{\mathrm{p}+\mathrm{k}}{2}\right) !}}{\left(\frac{\mathrm{p}-\mathrm{k}}{2}\right) !\left(\frac{\mathrm{k}+\mathrm{q}}{2}\right) !\left(\frac{\mathrm{k}-\mathrm{q}}{2}\right) !}$ & \\
$\begin{array}{l}\text { Zernike (Hosny, 2008) } \\
\begin{array}{l}\text { Pseudo Zernike } \\
\text { (Al-Rawi, 2010) }\end{array}\end{array}$ & $\begin{array}{l}\mathrm{T}=\mathrm{q} \text { \& \& } \\
(\mathrm{p}-\mathrm{q}) \text { is even }\end{array}$ \\
$\begin{array}{l}\text { Fourier-Mellin } \\
\text { (Papakostas et al., 2007) }\end{array}$ & $\frac{(-1)^{(\mathrm{p}-\mathrm{k})}(\mathrm{p}+\mathrm{k}+1) !}{(\mathrm{p}-\mathrm{k}) !(\mathrm{k}+\mathrm{q}+1) !(\mathrm{k}-\mathrm{q}) !}$ & $\mathrm{T}=\mathrm{q}$ \\
\hline
\end{tabular}

\begin{tabular}{|c|c|c|c|c|c|c|c|}
\hline 47 & 46 & 45 & 44 & 43 & 42 & 41 & 40 \\
\hline 48 & 24 & 23 & 22 & 21 & 20 & 19 & 39 \\
\hline 49 & 25 & 9 & 8 & 7 & 6 & 18 & 38 \\
\hline 50 & 26 & 10 & 2 & 1 & 5 & 17 & 37 \\
\hline 51 & 27 & 11 & 3 & 4 & 16 & 36 & 64 \\
\hline 52 & 28 & 12 & 13 & 14 & 15 & 35 & 63 \\
\hline 53 & 29 & 30 & 31 & 32 & 33 & 34 & 62 \\
\hline 54 & 55 & 56 & 57 & 58 & 59 & 60 & 61 \\
\hline
\end{tabular}

Fig. 1: Original square image pixels

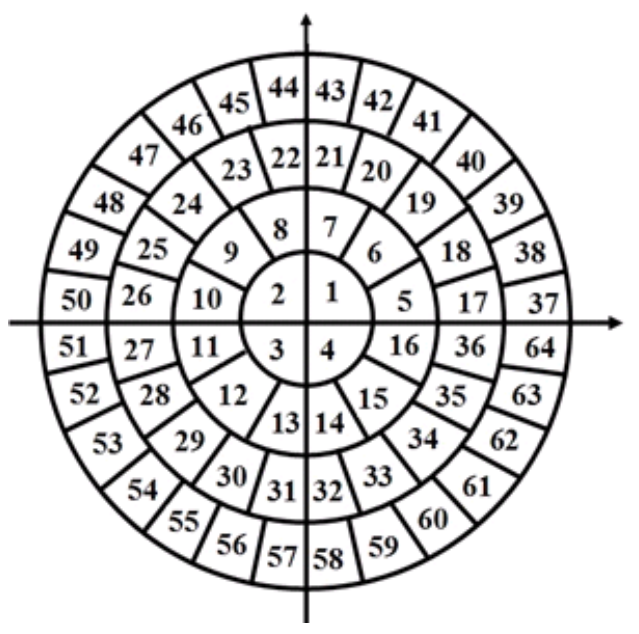

Fig. 2: Mapped circular image pixels 
The radial moments of order $\mathrm{k}$ and repetition $\mathrm{q}$ are:

$\mathrm{R}_{\mathrm{kq}}=\sum_{\mathrm{i}} \sum_{\mathrm{j}} \mathrm{f}\left(\mathrm{r}_{\mathrm{i}}, \theta_{\mathrm{i}, \mathrm{j}}\right) \mathrm{H}_{\mathrm{pq}}\left(\mathrm{r}_{\mathrm{i}}, \theta_{\mathrm{i}, \mathrm{j}}\right)$

With:

$$
H_{k q}\left(r_{i}, \theta_{i, j}\right)=I_{k}\left(r_{i}\right) I_{q}\left(\theta_{i j}\right)
$$

Where:

$$
\begin{aligned}
& I_{k}\left(r_{i}\right)=\int_{U_{i}}^{U_{i+1}} r^{k} r d r \\
& I_{q}\left(\theta_{i j}\right)=\int_{V_{i, j}}^{V_{i, j+1}} e^{-\hat{i} q \theta} d \theta
\end{aligned}
$$

Both integrals in Eq. 12 and 13 are evaluated analytically without any kind of approximation as follows:

$$
\begin{aligned}
& I_{k}\left(r_{i}\right)=\left(\frac{U_{i+1}{ }^{k+2}-U_{i}{ }^{k+2}}{p+2}\right) \\
& I_{q}\left(\theta_{i, j}\right)= \begin{cases}\frac{\hat{i}}{q}\left(e^{-\hat{i} q V_{i, j+1}}-e^{-\hat{i} q V_{i, j}}\right), q \neq 0 \\
V_{i, j+1}-V_{i, j} & , q=0\end{cases}
\end{aligned}
$$

The vectors of upper and lower limits could be defined as follows:

$$
\begin{aligned}
& U_{i+1}=\frac{(2 i+1)}{N} \\
& U_{i}=\frac{2 i}{N} \\
& V_{i, j+1}=\theta_{i, j}+\frac{\pi}{(8 i+4)} \\
& V_{i, j}=\theta_{i, j}-\frac{\pi}{(8 i+4)}
\end{aligned}
$$

It is clear that, the kernels, $\mathrm{I}_{\mathrm{k}}\left(\mathrm{r}_{\mathrm{i}}\right)$ and $\mathrm{I}_{\mathrm{q}}\left(\theta_{\mathrm{kj}}\right)$ are image-independent. Therefore, both kernels could be pre-computed and stored for any future use.

For efficient computation of highly accurate radial moments, one-dimensional cascade is implemented for fast computation and two types of symmetry properties are applied for memory reduction. Both of these approaches were successfully employed in the theme of moment computation.

Equation 10 will be rewritten in a separable form as follows:

$$
\begin{aligned}
& R_{k q}=\sum_{i} I_{k}\left(r_{i}\right) Y_{i q} \\
& Y_{i q}=\sum_{j} I_{q}\left(\theta_{i j}\right) f\left(r_{i}, \theta_{i j}\right)
\end{aligned}
$$

It must be noted that, an 8-way symmetry property could be applied to achieved $87 \%$ of memory saving. For detailed description of this symmetry, the readers are referred to the study (Hosny et al., 2011)

\section{RESULTS}

A number of numerical experiments are conducted to prove the validity and the efficiency of the proposed method. Two standard gray level images as displayed in Fig. 3 are used where the full set of Zernike and orthogonal Fourier-Mellin are computed by using the proposed method and the traditional approximated ZOA methods. Mean Square Error (MSE) is used in the evaluation process. The computed sets of moments are used to reconstruct the input image and then, the MSE are computed. The values of the MSE are plotted.

Two numerical experiments are conducted. In the first experiment, the 'house' gray level image of size $128 \times 128$ is used, where the full set of orthogonal Fourier-Mellin moments up to order $\operatorname{Max}=20$ is computed by using the proposed method and the ZOA method. The MSE of both methods are computed, plotted and depicted in Fig. 4. It is clear that, the MSE of the proposed method decreased as the moment order increased. On the other side, the MSE of the ZOA method decreased for few moment orders and suddenly increased. This is evidence on the accuracy of the proposed method.
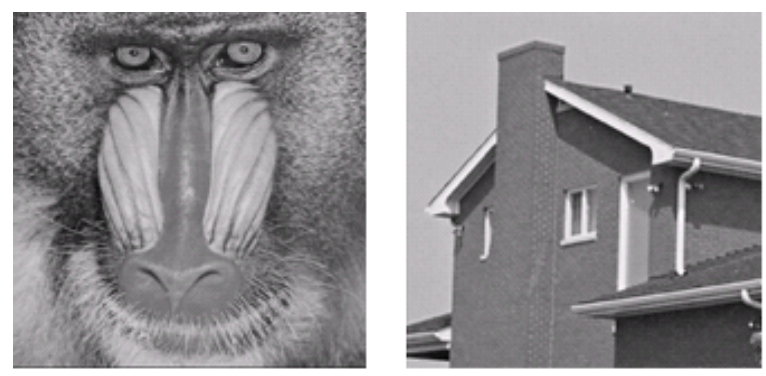

Fig. 3: Standard gray level images 


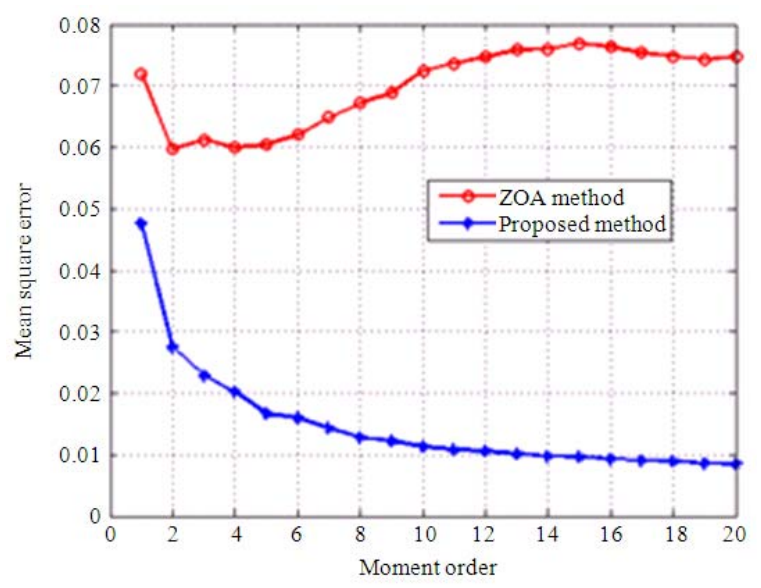

Fig. 4: Mean square errors: Orthogonal Fourier-Mellin moments

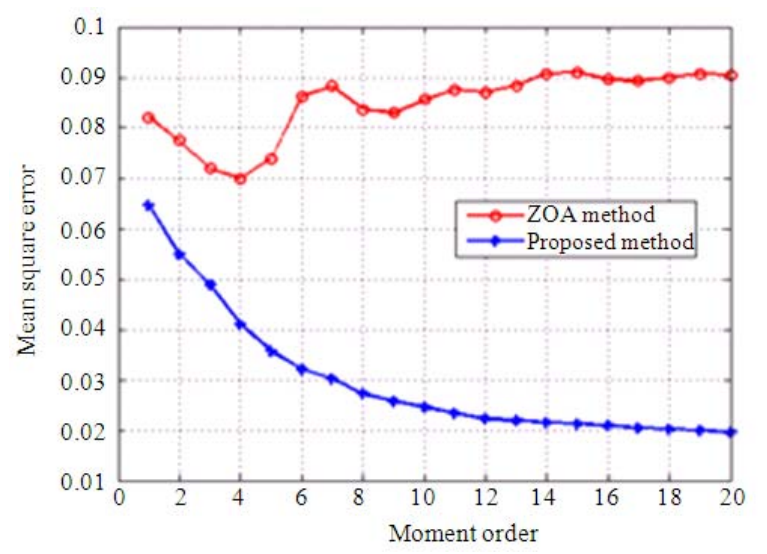

Fig. 5: Mean square errors: Zernike moments

In the second experiment, the full set of Zernike moments are computed with the proposed and the ZOA method. The MSE of both methods are computed, plotted and depicted in Fig. 5. As expected, a similar result is reached.

Since pseudo Zernike moments are derived from original Zernike moments and based on the big similarity between their functions, the computational process of pseudo Zernike moments is straightforward.

All computational processes are performed by using a code designed with Matlab8 and operated on a Lenovo R400 Laptop.

Invariance to different kinds of geometric transformations such as rotation, translation and scale will be tested. Invariance of the Zernike, pseudo Zernike and Fourier-Mellin moments are tested using standard gray level images.

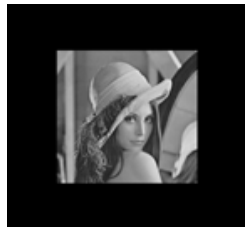

(a)

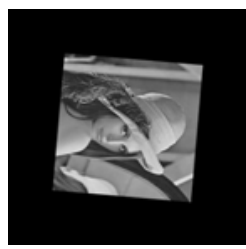

(d)

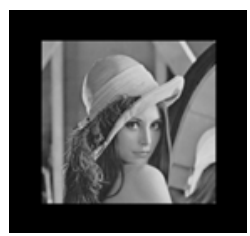

(g)

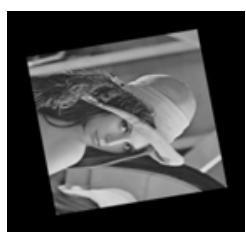

(j)

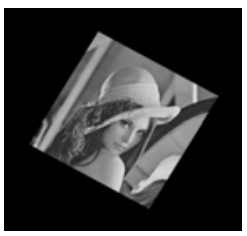

(b)

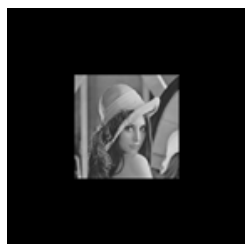

(e)

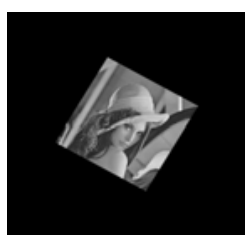

(h)

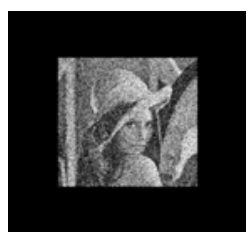

(k)

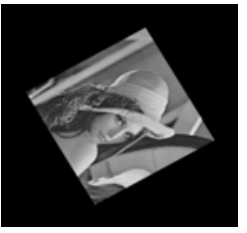

(c)

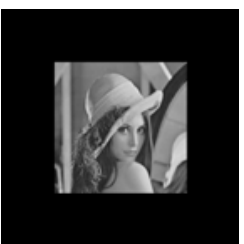

(f)

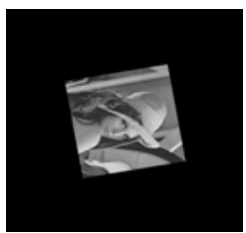

(i)

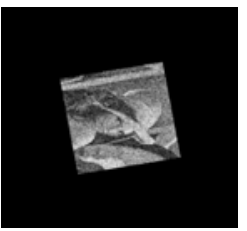

(l)
Fig. 6: (a) Original image, (b) Rotation $\mathrm{R}=30^{\circ}$, (c) $\mathrm{R}=$ $60^{\circ}$ and (d) $\mathrm{R}=95^{\circ}$, (e) Scaling $\mathrm{S}=0.75$, (f) $\mathrm{S}=$ 0.95 , (g) $\mathrm{S}=1.25$, (h) $\mathrm{S}=0.75$ and $\mathrm{R}=30^{\circ}$, (i) $\mathrm{S}$ $=0.75$ and $\mathrm{R}=80^{\circ},(\mathrm{j}) \mathrm{S}=1.25$ and $\mathrm{R}=80^{\circ}$, $(\mathrm{k})$ Noisy image, (l) $\mathrm{S}=0.75$ and $\mathrm{R}=80^{\circ}$

These numerical experiments were designed in (Hosny, 2008). The first experiment is concerned with testing invariance against rotation where the original gray level image of Lena is rotated by $30^{\circ}, 60^{\circ}$ and $95^{\circ}$ as shown in Fig. 6b-d.

The absolute errors between the low order orthogonal moments of the original image and the rotated ones are showed in Table 2-4 for Zernike, pseudo Zernike and Fourier-Mellin moment invariants respectively. It is clear that, the absolute errors are very small whatever the rotation angle which ensure the accuracy of the proposed unified method.

Invariance against scaling for both image reduction and enlargement is tested. The image of 'Lena' is reduced by the scaling factors 0.75 and 0.95 . It is also enlarged by the factor 1.25 as shown in Fig. 6e-f and $7 \mathrm{~g}$. The absolute errors of the low order moments for original and the scaled images are showed. As expected, the absolute errors of the proposed moment invariants are very small. 
J. Computer Sci., 7 (5): 715-722, 2011

Table 2: Absolute values of low order Zernike moments (First experiment)

\begin{tabular}{|c|c|c|c|c|c|c|c|c|c|}
\hline \multicolumn{3}{|c|}{ Transformation } & \multicolumn{7}{|c|}{ Zernike moments } \\
\hline $\mathrm{R}$ & S & $\mathrm{N}$ & $\mathrm{X}_{20}$ & $\mathrm{Z}_{22}$ & $\mathrm{Z}_{31}$ & $\mathrm{Z}_{33}$ & $\mathrm{Z}_{40}$ & $\mathrm{Z}_{42}$ & $\mathrm{Z}_{44}$ \\
\hline 300 & ----- & 0 & 0.0787 & 0.0354 & 0.0348 & 0.039 & 0.0417 & 0.0143 & 0.0297 \\
\hline 600 & ----- & 0 & 0.0784 & 0.0351 & 0.0345 & 0.0392 & 0.0415 & 0.0141 & 0.0296 \\
\hline 950 & ----- & 0 & 0.0786 & 0.0354 & 0.0347 & 0.0391 & 0.0416 & 0.0140 & 0.0293 \\
\hline ----- & 0.75 & 0 & 0.0787 & 0.0354 & 0.0348 & 0.0390 & 0.0417 & 0.0143 & 0.0297 \\
\hline ------ & 0.95 & 0 & 0.0786 & 0.0354 & 0.0348 & 0.0390 & 0.0418 & 0.0143 & 0.0297 \\
\hline ----- & 1.25 & 0 & 0.0787 & 0.0353 & 0.0347 & 0.0388 & 0.0417 & 0.0143 & 0.0297 \\
\hline 800 & 0.75 & 0 & 0.0787 & 0.0352 & 0.0346 & 0.0390 & 0.0414 & 0.0143 & 0.0296 \\
\hline 800 & 1.25 & 0 & 0.0783 & 0.0350 & 0.0348 & 0.0386 & 0.0417 & 0.0141 & 0.0296 \\
\hline 800 & 0.75 & 1 & 0.0778 & 0.0343 & 0.0344 & 0.0392 & 0.0422 & 0.0138 & 0.0283 \\
\hline
\end{tabular}

Table 3: Absolute values of low order pseudo Zernike moments (First experiment)

Transformation Pseudo Zernike moments

\begin{tabular}{|c|c|c|c|c|c|c|c|c|c|c|}
\hline & \\
\hline$\underline{\mathrm{R}}$ & $\mathrm{S}$ & $\mathrm{N}$ & $\mathrm{A}_{20}$ & $\mathrm{~A}_{21}$ & $\mathrm{~A}_{22}$ & $\mathrm{~A}_{31}$ & $\mathrm{~A}_{32}$ & $\mathrm{~A}_{33}$ & $\mathrm{~A}_{42}$ & $\mathrm{~A}_{44}$ \\
\hline 300 & ----- & 0 & 0.1827 & 0.0347 & 0.0185 & 0.0648 & 0.0144 & 0.0221 & 0.0251 & 0.1200 \\
\hline 600 & ----- & 0 & 0.1825 & 0.0345 & 0.0185 & 0.0647 & 0.0144 & 0.0223 & 0.0250 & 0.1201 \\
\hline 950 & ----- & 0 & 0.1824 & 0.0343 & 0.0184 & 0.0645 & 0.0145 & 0.0224 & 0.0253 & 0.1203 \\
\hline ----- & 0.75 & 0 & 0.1827 & 0.0347 & 0.0185 & 0.0648 & 0.0144 & 0.0221 & 0.0251 & 0.1200 \\
\hline ----- & 0.95 & 0 & 0.1827 & 0.0347 & 0.0185 & 0.0648 & 0.0144 & 0.0221 & 0.0251 & 0.1200 \\
\hline ----- & 1.25 & 0 & 0.1826 & 0.0347 & 0.0185 & 0.0647 & 0.0144 & 0.0222 & 0.0251 & 0.1200 \\
\hline 800 & 0.75 & 0 & 0.1827 & 0.0345 & 0.0185 & 0.0648 & 0.0145 & 0.0221 & 0.0252 & 0.1202 \\
\hline 800 & 1.25 & 0 & 0.1825 & 0.0347 & 0.0183 & 0.0645 & 0.0144 & 0.0223 & 0.0253 & 0.1201 \\
\hline 800 & 0.75 & 1 & 0.1819 & 0.0339 & 0.0181 & 0.0641 & 0.0149 & 0.0216 & 0.0245 & 0.1204 \\
\hline
\end{tabular}

Table 4: Absolute values of low order orthogonal Fourier-Mellin moments (First experiment) Transformation Orthogonal Fourier-Mellin moments

\begin{tabular}{|c|c|c|c|c|c|c|c|c|c|c|}
\hline $\mathrm{R}$ & $\mathrm{S}$ & $\mathrm{N}$ & $\mathrm{F}_{20}$ & $\mathrm{~F}_{21}$ & $\mathrm{~F}_{22}$ & $\mathrm{~F}_{30}$ & $\mathrm{~F}_{31}$ & $\mathrm{~F}_{32}$ & $\mathrm{~F}_{33}$ & $\mathrm{~F}_{42}$ \\
\hline 300 & ----- & 0 & 0.0443 & 0.0155 & 0.0101 & 0.0139 & 0.0074 & 0.0112 & 0.0235 & 0.0043 \\
\hline 600 & ----- & 0 & 0.0442 & 0.0156 & 0.0101 & 0.0138 & 0.0072 & 0.0111 & 0.0234 & 0.0044 \\
\hline 950 & ----- & 0 & 0.0441 & 0.0154 & 0.0103 & 0.0137 & 0.0075 & 0.0114 & 0.0233 & 0.0043 \\
\hline ----- & 0.75 & 0 & 0.0443 & 0.0155 & 0.0101 & 0.0139 & 0.0074 & 0.0112 & 0.0235 & 0.0043 \\
\hline ----- & 0.95 & 0 & 0.0443 & 0.0155 & 0.0101 & 0.0139 & 0.0074 & 0.0111 & 0.0233 & 0.0043 \\
\hline ----- & 1.25 & 0 & 0.0444 & 0.0154 & 0.0102 & 0.0137 & 0.0073 & 0.0112 & 0.0235 & 0.0042 \\
\hline 800 & 0.75 & 0 & 0.0441 & 0.0155 & 0.0101 & 0.0138 & 0.0075 & 0.0112 & 0.0232 & 0.0043 \\
\hline 800 & 1.25 & 0 & 0.0441 & 0.0153 & 0.0101 & 0.0139 & 0.0074 & 0.0112 & 0.0235 & 0.0042 \\
\hline 800 & 0.75 & 1 & 0.0438 & 0.0150 & 0.0094 & 0.0132 & 0.0064 & 0.0107 & 0.0239 & 0.0035 \\
\hline
\end{tabular}

To test robustness against noise, a white Gaussian noise is added to the image of Lena according to the MATLAB statement; $A=$ imnoise (A, 'Gaussian', 0, 0.05). The noisy image (Fig. 6k) is scaled with the factor 0.75 and then rotated with the angle $80^{\circ}$ (as displayed in Fig. 6l).

Another group of numerical experiments are conducted where the image of recycling logo is used. These numerical experiments were designed in (Hosny, 2008) where the input test image is 3-fold rotation symmetry. The gray level image of recycle logo with size $128 \times 128$ (Fig. 7a) is rotated by $30^{\circ}, 60^{\circ}$ and $95^{\circ}$ as shown in Fig. 7b-d. As in the previous numerical experiments, the absolute errors of low order orthogonal moments are computed and shown in Table 5.

Invariance against a combination of scaling and rotation is tested where the test image is reduced by the percentage of $75 \%$ and then rotated through the angle $80^{\circ}$. In another experiment, the test image is enlarged using the percentage $125 \%$ and then rotated through the angle $80^{\circ}$ as shown in Fig. 7e and $\mathrm{f}$.

Robustness against the white Gaussian noise is tested. The noisy image (Fig. 7g) is scaled with the factor 0.75 and then the noisy image is rotated by an angle $30^{\circ}$ (Fig. 7h). The absolute errors are showed in Table 5. The acronyms ' $\mathrm{R}$ ' and ' $\mathrm{S}$ ' refer to rotation and scaling respectively. The values ' 0 ' means noise-free while ' 1 ' means noisy.

The conducted experiments clearly show the accuracy of the proposed unified method. All aspects of accuracy evaluation such as image reconstruction, invariance with respect to geometric transformations (rotation and scaling) and robustness against noise are considered. The proposed unified method is proved to be very effective in case of symmetric images. 
Table 5: Absolute values of low order Zernike moments (Second experiment)

\begin{tabular}{|c|c|c|c|c|c|c|c|c|c|}
\hline \multicolumn{3}{|c|}{ Transformation } & \multicolumn{7}{|c|}{ Zernike moments } \\
\hline $\mathrm{R}$ & $\mathrm{S}$ & $\mathrm{N}$ & $\mathrm{Z}_{20}$ & $\mathrm{Z}_{22}$ & $\mathrm{Z}_{31}$ & $\mathrm{Z}_{33}$ & $\mathrm{Z}_{40}$ & $\mathrm{Z}_{42}$ & $\mathrm{Z}_{44}$ \\
\hline 300 & ---- & 0 & 0.0839 & 0.1382 & 0.0394 & 0.0844 & 0.5987 & 0.0336 & 0.0108 \\
\hline 600 & ---- & 0 & 0.0838 & 0.1381 & 0.0392 & 0.0842 & 0.5985 & 0.0335 & 0.0106 \\
\hline 950 & ---- & 0 & 0.0835 & 0.1385 & 0.0390 & 0.0841 & 0.5986 & 0.0337 & 0.0102 \\
\hline 800 & 0.75 & 0 & 0.0832 & 0.1381 & 0.0391 & 0.0842 & 0.5986 & 0.0334 & 0.0107 \\
\hline 800 & 1.25 & 0 & 0.0832 & 0.1381 & 0.0391 & 0.0842 & 0.5986 & 0.0334 & 0.0107 \\
\hline 300 & 0.75 & 1 & 0.0839 & 0.1382 & 0.0393 & 0.0844 & 0.5987 & 0.0336 & 0.0108 \\
\hline
\end{tabular}

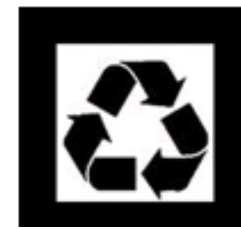

(a)

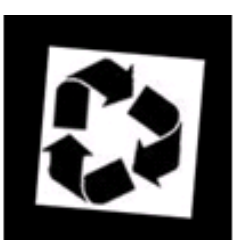

(d)

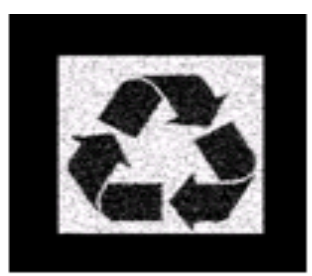

(g)

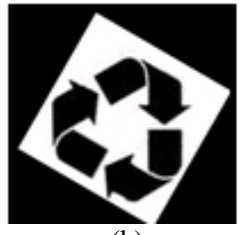

(b)

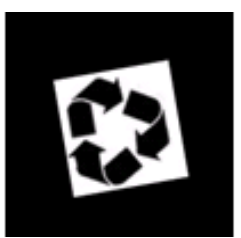

(e)

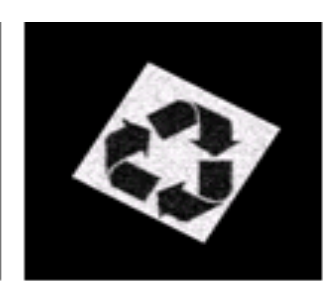

(h)

(c)

(f)
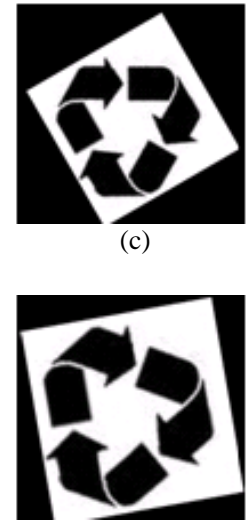

Fig. 7: (a) recycling symbol's image, (b) Rotation $\mathrm{R}=$
$30^{\circ}$, (c) $\mathrm{R}=60^{\circ}$, (d) $\mathrm{R}=95^{\circ}$, (e) $\mathrm{S}=0.75$ and $\mathrm{R}$ $=80^{\circ}$, (f) $\mathrm{S}=1.25$ and $\mathrm{R}=80^{\circ}$, (g) Noisy image, (h) Noise and $\mathrm{S}=0.75$ and $\mathrm{R}=30^{\circ}$

\section{DISCUSSION}

The conducted experiments clearly show the accuracy of the proposed unified method. All aspects of accuracy evaluation such as image reconstruction, invariance with respect to geometric transformations (rotation and scaling) and robustness against noise are considered. The proposed unified method is proved to be very effective in case of symmetric images.

\section{CONCLUSION}

This study proposes a unified method for accurate computation of circular moments for gray-scale images. A new image mapping approach is proposed. In this approach, the total number of the Cartesian pixels of the input digital image is equal to the corresponding circular image pixels in polar coordinates. Radial moments are accurately computed by using the proposed mapping scheme and then; Zernike, pseudo Zernike and Fourier-Mellin moments are expressed as a linear combination of radial moments. The numerical experiments are performed for real images with different sizes to ensure the efficiency of the proposed method.

\section{ACKNOWLEDGMENT}

Dr. Khalid M. Hosny gratefully acknowledges the financial support of Najran University (Grant No. NU24/2010).

\section{REFERENCES}

Abandah, G. and N. Anssari, 2009. Novel moment features extraction for recognizing handwritten Arabic letters. J. Comput. Sci., 5: 226-232. ISSN: 1549-3636

Al-Rawi, M.S., 2010. Fast computation of pseudo Zernike moments. J. Real-Time Image Proc., 5: 310. DOI: $10.1007 / \mathrm{s} 11554-009-0118-0$

Chong, C.W., R. Paramesan and R. Mukundan, 2003a. An efficient algorithm for fast computation of pseudo-Zernike moments. Int. J. Pat. Recog. Arti. Intell, 17 : 1011-1023. 10.1142/S0218001403002769

Chong, C.W., P. Raveendran and R. Mukundan, 2003b. Translation invariants of Zernike moments. Pat. Recog., 36: 1765-1773. DOI: 10.1016/S00313203(02)00353-9

Chong, C.W., P. Raveendran and R. Mukundan, 2003c. The scale invariants of pseudo-Zernike moments. Pat. Anal. Appl., 6: 176-184. DOI: 10.1007/s10044-002-0183-5

Hosny, K.M., 2008. Fast computation of accurate Zernike moments. J. Real-Time Image Proc., 3: 97107. DOI: $10.1007 /$ s11554-007-0058-5

Hosny, K.M., 2008. Optimized set of RST moment invariants. Am. J. Applied Sci., 5: 726-735. DOI: 10.3844/ajassp.2008. 726.735 
Hosny, K.M., 2010a. Fast and accurate method for radial's moments computation. Pat. Recog. Lett., 31: 143-150. DOI: 10.1016/j.patrec.2009.09.027

Hosny, K.M., 2010b. A systematic method for efficient computation of full and subsets Zernike moments. Inform. Sci., 180: 2299-2313. DOI: 10.1016/j.ins.2010.02.006

Hosny, K.M., M.A. Shouman and H.M Abdel-Salam, 2011. Fast computation of orthogonal FourierMellin moments in polar coordinates. J . Real-Time Image Proc. DOI: 10.1007/s11554-009-0135-z

Ismail, I.A., M.A. Shouman, K.M. Hosny and Abdel H.M. Salam, 2010. Invariant image watermarking using accurate Zernike moments. J. Comput. Sci., 6: 52-59.

Papakostas, G.A., Y.S, Boutalis, D.A. Karras and B.G. Mertzios, 2007. Fast numerically stable computation of orthogonal fourier-mellin moments. IET Comput. Vis., 1: 11-16. DOI: 10.1049/ietcvi:20060130
Radhika, K.R., M.K. Venkatesha and G.N. Sekhar, 2010. Off-line signature authentication based on moment invariants using support vector machine. J. Comput. Sci., 6: 305-311.

Walia, E., C. Singh and A. Goyal, 2011. On the fast computation of orthogonal Fourier-Mellin moments with improved numerical stability. J. Real-Time Image Proc. DOI: 10.1007/s11554-0100172-7

Xin, Y., M. Pawlak and S. Liao, 2007. Accurate computation of Zernike moments in polar coordinates. IEEE Trans. Image Proc. 16: 581-587. DOI: 10.1109/TIP.2006.888346 